\title{
Prevalência de enteroparasitas em crianças provenientes da área rural de Cáceres-
}

\section{MT}

\author{
Prevalence of endoparasites in children from the rural area of Cáceres-MT \\ Prevalencia de enteroparasitos en niños del área rural de Cáceres-MT
}

Recebido: 19/11/2021 | Revisado: 29/11/2021 | Aceito: 30/11/2021 | Publicado: 07/12/2021

Luan Marcelo Gonçalves Leite

ORCID: https://orcid.org/0000-0001-9213-1953 Universidade do Estado de Mato Grosso, Brasil

E-mail: luan.marcelo@unemat.br

Denise da Costa Boamorte Cortela

ORCID: https://orcid.org/0000-0003-0052-6216 Universidade do Estado de Mato Grosso, Brasil

E-mail: denisecortela@hotmail.com

Lucas França de Barros

ORCID: https://orcid.org/0000-0001-5203-178X Universidade do Estado de Mato Grosso, Brasil E-mail: lucasmariano.f@gmail.com

Geainny Gomes de Souza

ORCID: https://orcid.org/0000-0003-2660-5318 Universidade do Estado de Mato Grosso, Brasil E-mail: geainny.gomes@unemat.com.br

Andernice dos Santos Zanetti

ORCID: https://orcid.org/0000- 0001-7888-8183 Universidade do Estado de Mato Grosso, Brasil E-mail: andernice@gmail.com

Marta dos Santos Miranda de Araújo

ORCID: https://orcid.org/0000-0002-3165-8230 Universidade do Estado de Mato Grosso, Brasil E-mail: martamiranda2@hotmail.com

Antonio Francisco Malheiros

ORCID: https://orcid.org/0000-0001-8169-0557 Universidade do Estado de Mato Grosso, Brasil E-mail: malheiros@unemat.br

\begin{abstract}
Resumo
As enteroparasitas são comuns em indivíduos que se encontram sob condições precárias de moradia, água potável, saneamento básico e higiene. $\mathrm{O}$ objetivo deste trabalho é verificar a prevalência de enteroparasitas em crianças em fase escolar do assentamento/comunidade Paiol, no município de Cáceres-MT. Análises parasitológicas pelo método de Hoffman, além dos dados socioeconômicos e ambientais foram realizadas em 75 amostras de crianças com idade de 7 a 16 anos. A prevalência geral de enteroparasitas foi de 56\% na população estudada. Protozoários foram identificados em $98 \%$ das amostras positivas sendo Blastocystis hominis o mais prevalente seguido de Endolimax nana, Entamoeba coli e Giardia lamblia. Helmintos foram identificados em 5\% do total de amostras analisadas, sendo Trichuris trichiura e Hymenolepis nana os únicos identificados. Conclui-se que há elevada prevalência de enteroparasitas nas crianças, principalmente de protozoários intestinais, sendo necessária melhorias e monitoramento das condições higiênico-sanitárias em comunidades rurais da região estudada.
\end{abstract}

Palavras-chave: Parasitas intestinais; Assentamento rural; Pantanal mato-grossense.

\begin{abstract}
Enteroparasites are common in individuals who are under precarious conditions of housing, cleans water, basic sanitation and hygiene. The objective of this study is to verify the prevalence of intestinal parasitosis in school-age children from the Paiol settlement/community, in the city of Cáceres-MT. Parasitological analyzes using the Hoffman method, in addition to socioeconomic and environmental data, were performed in 75 samples of children aged 7 to 16 years. The overall prevalence of intestinal parasites was 56\% in the studied population. Protozoa were identified in 98\% of positive samples, with Blastocystis hominis being the most prevalent, followed by Endolimax nana, Entamoeba coli, and Giardia lamblia. Helminths were identified in 5\% of the total samples analyzed, with Trichuris trichiura and Hymenolepis nana being the only ones identified. It is concluded that there is a high prevalence of intestinal parasite in children, especially of intestinal protozoa, requiring improvements and monitoring of hygienicsanitary conditions in rural communities in the studied region.
\end{abstract}

Keywords: Intestinal parasites; rural settlement; Mato Grosso Pantanal. 


\begin{abstract}
Resumen
Los enteroparásitos son comunes en personas que se encuentran en condiciones precarias de vivienda, agua potable, saneamiento básico e higiene. El objetivo de este estudio es verificar la prevalencia de parásitos intestinales en niños en edad escolar del asentamiento / comunidad Paiol, en Cáceres-MT. Se realizaron análisis parasitológicos mediante el método de Hoffman, además de datos socioeconómicos y ambientales, en 75 muestras de niños de 7 a 16 años. La prevalencia global de parásitos intestinales fue del $56 \%$ en la población estudiada. Se identificaron protozoos en el 98\% de las muestras positivas, siendo Blastocystis hominis el más prevalente, seguido de Endolimax nana, Entamoeba coli y Giardia lamblia. Se identificaron helmintos en el 5\% del total de muestras analizadas, siendo Trichuris trichiura e Hymenolepis nana los únicos identificados. Se concluye que existe una alta prevalencia de parásitos intestinales en niños, especialmente protozoos intestinales, requiriendo mejoras y monitoreo de las condiciones higiénico-sanitarias en las comunidades rurales de la región estudiada.
\end{abstract}

Palabras clave: Parasitosis intestinal; Asentamiento rural; Pantanal de Mato Grosso.

\title{
1. Introdução
}

As enteroparasitoses são infecções causadas por parasitas intestinais, sendo em sua maioria desencadeadas por protozoários intestinais e helmintos. Os protozoários mais predominantes em humanos são os Cryptosporidium sp., Endolimax nana, Entamoeba coli, Entamoeba histolytica e Giardia lamblia. Entre os helmintos temos Ascaris lumbricoides, Enterobius vermicularis, Trichuris trichiura, e ancilostomídeos. (Andrade et al., 2013; OMS, 2013).

Os enteroparasitas consistem em um problema socioeconômico relevante, principalmente nas regiões subdesenvolvidas e em desenvolvimento, estando normalmente ligada a falta de saneamento básico e na escassez de higiene, contribuindo desta forma, na manutenção do ciclo biológico e na disseminação dos parasitas. (Silva et al., 2018).

Estima-se que 200 milhões de crianças na idade pré-escolar de todo o mundo sejam afetadas por enteroparasitas, devido à falta principalmente de estratégias de melhoramento do nível socioeconômico da população. (Muñoz-Antoli et al., 2018). No Brasil é alta a prevalência em crianças em idade escolar, a infecção por essas enteroparasitoses prejudica seu desenvolvimento físico, afetando o estado nutricional e consequentemente o rendimento escolar. (Barbosa et al., 2017; Souza, 2018).

Os indivíduos albergados por parasitas intestinais, podem se apresentar assintomáticos, principalmente nos estágios iniciais da infecção, o que os tornam importantes disseminadores. A transmissão que é oral-fecal ocorre, principalmente, através da ingestão de água ou alimentos contaminados por ovos embrionados, cistos ou larvas. (Mergulhão, 2020).

Os assentamentos rurais, devido ao vagaroso processo de investimento público para construção de infraestrutura adequada de moradia e saneamento básico, são potenciais focos da manutenção do ciclo da transmissão de doenças enteroparasitárias. (INCRA, 2021). Os assentamentos rurais tiveram maior visibilidade com o início do movimento de reforma agrária em meados dos anos 1990, e atualmente, há mais de 9 mil assentamentos nas regiões brasileiras. (INCRA, 2021; Girardi, Fernandes, 2008). Em seu estudo Viana et al. (2017) amostrou prevalência de 53,9\% em uma comunidade rural em Rosápolis de Paraíba- PI, associando as taxas de infecções enteroparasitas com condições higiênico-sanitárias precárias, as quais estão submetidos os indivíduos parasitados.

O Brasil está entre os países com maior índice de parasitoses intestinais, sendo alta a prevalência em crianças de idade pré-escolar, devido aos hábitos inadequados, falta da sensibilização dos pais e professores e a falha da prática de higiene. (Zanotto et al., 2018).

Santos et al. (2017) comentam que, os índices elevados de morbidade e mortalidade, especialmente na idade infantil, estão amplamente associados à pobreza e nutrição inadequada, falta de abastecimento de água tratada e rede de esgoto, diminuindo gradativamente nas classes economicamente mais elevadas, associado ao nível de instrução educacional.

Em crianças, os fatores associados às enteroparasitoses se intensificam, ficando diretamente relacionados com hábitos precários de higiene. A criança inicia o desenvolvimento das coordenações motoras e adquire às poucas habilidades de pegar objetivos, levando-os à boca como forma de exploração e reconhecimento do mundo que o cerca. (Astudillo \& Brava, 2017). 
Segundo Fonseca et al. (2017), a vulnerabilidade das crianças em idade escolar e parasitoses relaciona-se normalmente com a falta de higienização, mudanças comportamentais, desenvolvimento e mobilidade, possibilitando a interação com o meio ambiente, estando diretamente desta forma exposto a objetos contaminados e consequentemente aumentando a prevalência de infecções parasitárias nessa idade.

Esta pesquisa tem como objetivo verificar a prevalência de enteroparasitoses em crianças em fase escolar do assentamento/comunidade Paiol, no município de Cáceres-MT.

\section{Metodologia}

\subsection{Tipo de estudo}

A presente pesquisa trata-se de um estudo transversal, de abordagem quantitativa de amostra não probabilística, com levantamento enteroparasitológico em crianças em idade escolar da escola Paulo Freire na comunidade Paiol pertencente ao município de Cáceres-MT.

Segundo Hochman (2005) os estudos transversais descrevem uma situação representado pela doença. Então as pesquisas transversais podem ser usadas para prevalência, investigando doenças em grupos específicos, sendo casos novos e antigos em um determinado tempo e local. (Bordalo, 2006).

\subsection{Descrição do local de estudo}

O levantamento enteroparasitológico nas crianças foi realizado na Escola Municipal Paulo Freire (EMPF), na comunidade Paiol, localizada em uma área rural de Cáceres no estado de Mato Grosso.

A comunidade envolve uma área territorial de $158,80 \mathrm{~km}^{2}$ e localiza-se à $65 \mathrm{~km}$ do município Cáceres-MT. Essa comunidade foi criada no ano de 1997, formada por 221 famílias e seu território pertence a áreas do bioma Pantanal e Cerrado. (Brasil, 1982).

O clima, segundo classificação de Köppen, é tropical quente e úmido, apresentando estação chuvosa no verão e seca no inverno. O maior período de concentração pluvial média ocorre de dezembro a março e o período de maior estiagem ocorre de junho a agosto, apresentando precipitação total anual de $1.335 \mathrm{~mm}$. (Neves et al., 2016).

A cobertura vegetal da comunidade é caracterizada por sete coberturas vegetação, Área de Tensão Ecológica, Corpos D’Água, Floresta Aluvial, Pecuária com Presença de Vegetação Secundária, Savana Florestada com Presença de Savana Arborizada e Savana Gramíneo-Lenhosa. Ao longo dos anos de 1997 a 2013, conclui-se que grande parte da vegetação foi suprimida para dar espaço para agropecuária. (Silva et al., 2018).

Em seu estudo na comunidade Paiol Silva et al (2018) relatou que maior parte das famílias faz o uso antrópico do solo para pecuária, principalmente na atividade leiteira e também com criação de animais (gado, galinha e porco) no local, contribuindo assim para a subsistência e geração de renda.

\subsection{Coleta de dados}

Para a pesquisa foi confeccionado e impresso um inquérito epidemiológico padronizado com dados do perfil demográfico, ambiental e de higiene pessoal dos participantes da pesquisa. O Termo de Consentimento Livre e Esclarecido (TALE) foram entregues para que os responsáveis legais assinassem, e um frasco coletor universal esterilizado para cada participante que concordou em participar da pesquisa. Os frascos, o TALE e o inquérito foram devidamente identificados com os dados dos alunos para que não houvesse repetição de amostras e outros dados (nome completo, sexo, idade).

Em conjunto com a direção da escola, foi agendando um dia para a coleta das amostras, o inquérito epidemiológico e o TALE. As amostras foram armazenadas em um isopor refrigerado, e encaminhado junto ao inquérito e o TALE para o 
LaBPar - Laboratório de Biologia Parasitária da Universidade do Estado de Mato Grosso, situado em Cáceres-MT, onde foram devidamente armazenados e as amostras refrigeradas a temperatura de $4{ }^{\circ} \mathrm{C}$ para posteriormente serem analisadas.

\subsection{Análises parasitológicas}

Para as análises parasitológicas, foi empregada a técnica coprológica de sedimentação espontânea em água, determinada por Hoffman et al. (1934).

Existem diversos métodos para a realização de exames parasitológicos, sendo que possuem especificidades e sensibilidades diferentes. (Cognialli, 2014). Das técnicas empregadas rotineiramente, o exame direto a fresco e a técnica de Hoffman, Pons e Janer (HOFFMAN, 1934) são métodos mais utilizados. Esta técnica possui como principal vantagem a necessidade mínima de materiais e recursos financeiros e que permite diagnosticar cistos de Protozoários, ovos e larvas de Helmintos. (Menezes, 2013).

Os resultados foram expressos como "POSITIVO e/ou NEGATIVO" para ovos/cistos e o nome do parasita identificado na análise. Os resultados positivos foram encaminhados para o médico que faz parte da equipe do projeto, para que houvesse o tratamento adequado das crianças em concordância com os pais, os resultados foram entregues para a direção da escola que assim entraria em contato com os pais/responsáveis.

\subsection{Aspectos éticos}

O Projeto foi aprovado pelo Comitê de Ética em Pesquisa com seres humanos sob o número do Parecer: 2.656.453 e CAAE: 83803318.4.0000.5166 em 15 de maio de 2018.

\section{Resultados e Discussões}

Neste estudo foram coletadas e analisadas 75 amostras fecais de crianças com idade de 7 a 16 anos, da (EMPF) na comunidade rural Paiol próximo do município de Cáceres - MT. Além das amostras coprológicas, foram coletados também, dados e parâmetros socioeconômicos e demográficos dos estudantes. Dentre as 75 amostras analisadas 60\% (45) correspondem ao sexo masculino e $40 \%$ (30) são do sexo feminino (Figura 1 a). Segregando as amostras por grupos de idade, $65 \%$ (49) são do grupo de 7 a 11 anos, sendo 35\% (26) do sexo masculino e 31\% (23) do sexo feminino. O grupo de 12 a 16 anos compreende 35\% (26) das amostras, sendo 17\% (13) para ambos sexos (Figura 1 b). 
Figura 1: Distribuição por sexo e idade das amostras analisadas da comunidade Paiol rural, Cáceres - Mato Grosso.

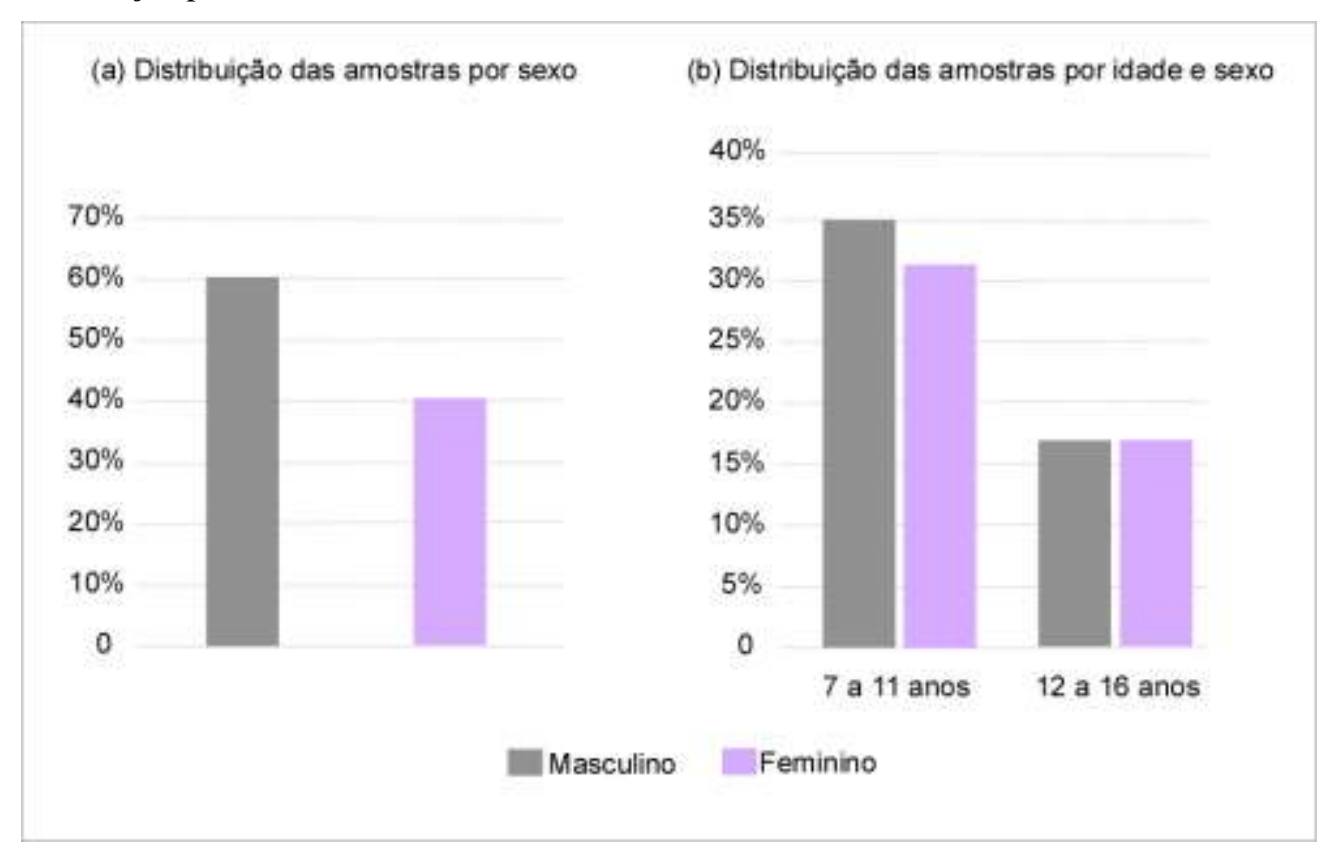

Fonte: Elaboração própria.

\subsection{Prevalência de infecções por enteroparasitas}

Das 75 amostras analisadas, identificamos que 56\% (42) estavam positivas para alguma espécie de parasita intestinal. As infecções por Protozoários foram as mais prevalentes, observadas em $98 \%$ das amostras positivas e as infecções por Helmintos, identificadas em apenas $5 \%$ das amostras positivas.

Crianças do sexo masculino foram as mais acometidas por enteroparasitas com 55\% (23) de prevalência, em comparação com as crianças do sexo feminino que apresentaram $45 \%$ (19) de prevalência (Figura 2).

Figura 2: Prevalência geral e por sexo de enteroparasitas nas amostras analisadas da comunidade Paiol rural, Cáceres - Mato Grosso.

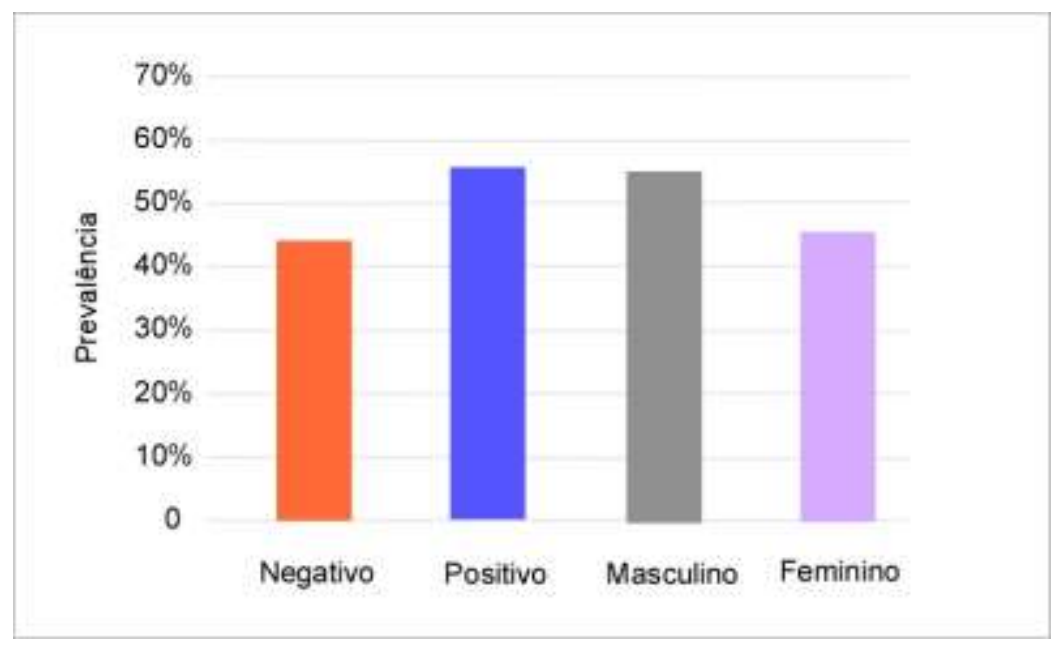

Fonte: Elaboração própria.

A prevalência geral de enteroparasitas foram similares aos estudos realizados em crianças de diversas regiões brasileiras, como Cardoso et al. (2020) que relatou uma prevalência geral de 39,2\% de infecções parasitárias em crianças de 03 a 10 anos de idade em três comunidades carentes em Teresinha-PI. Oliveira et al. (2019) determinaram em seus estudos 
prevalência geral de 55\% de crianças com idade de 6 e 12 anos em um município do Maranhão.

Viana et al. (2017) destaca que indivíduos residentes em povoamentos isolados, tais como comunidades rurais e assentamentos, estão entre os mais vulneráveis às contaminações por infecções parasitárias. As crianças são mais acometidas com enteroparasitas por causa da baixa condição higiênica sanitária e falta de amadurecimento do sistema imunológico. (Junior, 2020).

Em relação à faixa etária, as amostras foram analisadas por grupos das diferentes idades e foi observado que as idades de 15 e 16 anos apresentaram 100\% de prevalência de casos positivos, ressaltando que nesses dois grupos haviam uma e três amostras, respectivamente. Em sequência, os grupos com idade de 9 (quatorze amostras), 10 (oito amostras), 13 (dez amostras) e 11 anos (treze amostras), apresentaram 71\%,62\%, 54\% e 60\% de casos positivos, respectivamente. Os grupos de 7 (seis amostras) e 14 anos (duas amostras), apresentaram 50\% de casos positivos em ambas idades. Apenas as idades de 8 (oito amostras) e 12 anos (dez amostras), apresentaram menores porcentagens de casos positivos em comparação com os negativos, sendo 38\% e 30\%, respectivamente (Figura 3).

De um modo geral, a distribuição das prevalências enteroparasitárias nas diferentes idades das crianças analisadas variou de $30 \%$ a $100 \%$.

Figura 3: Prevalência geral de enteroparasitas por idade nas amostras analisadas na comunidade Paiol rural, Cáceres-Mato Grosso.

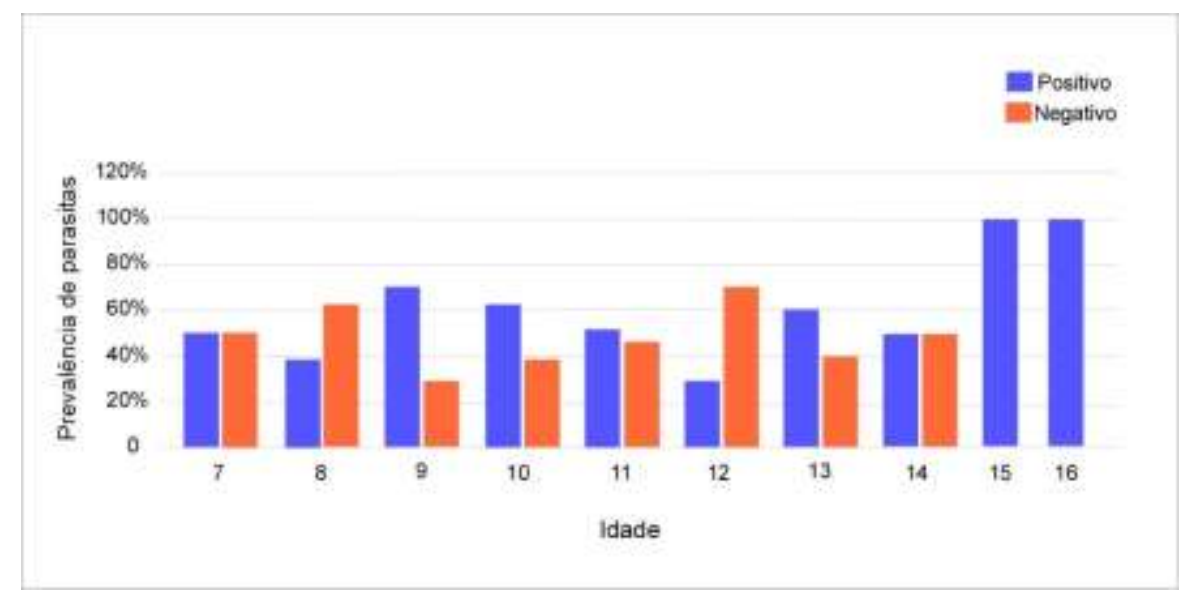

Fonte: Elaboração própria.

Foram observados a presença de seis Protozoários e dois Helmintos nas amostras coletadas. As análises identificaram Blastocystis hominis como o protozoário intestinal mais prevalente, observado em $71 \%$ (30) das amostras positivas, seguido de Endolimax nana em 29\% (12), Entamoeba coli em 26\% (11), Giardia lamblia em 19\% (8), Chilomastix mesnili em 7\% (3) e Entamoeba histolytica em 2\% (1) das amostras. Os Helmintos identificados foram Trichuris trichiura e Hymenolepis nana, ambos em 2\% (1) das amostras analisadas (Figura 4). 
Figura 4: Distribuição da prevalência dos parasitas intestinais observados nas amostras da comunidade Paiol rural, Cáceres Mato Grosso.

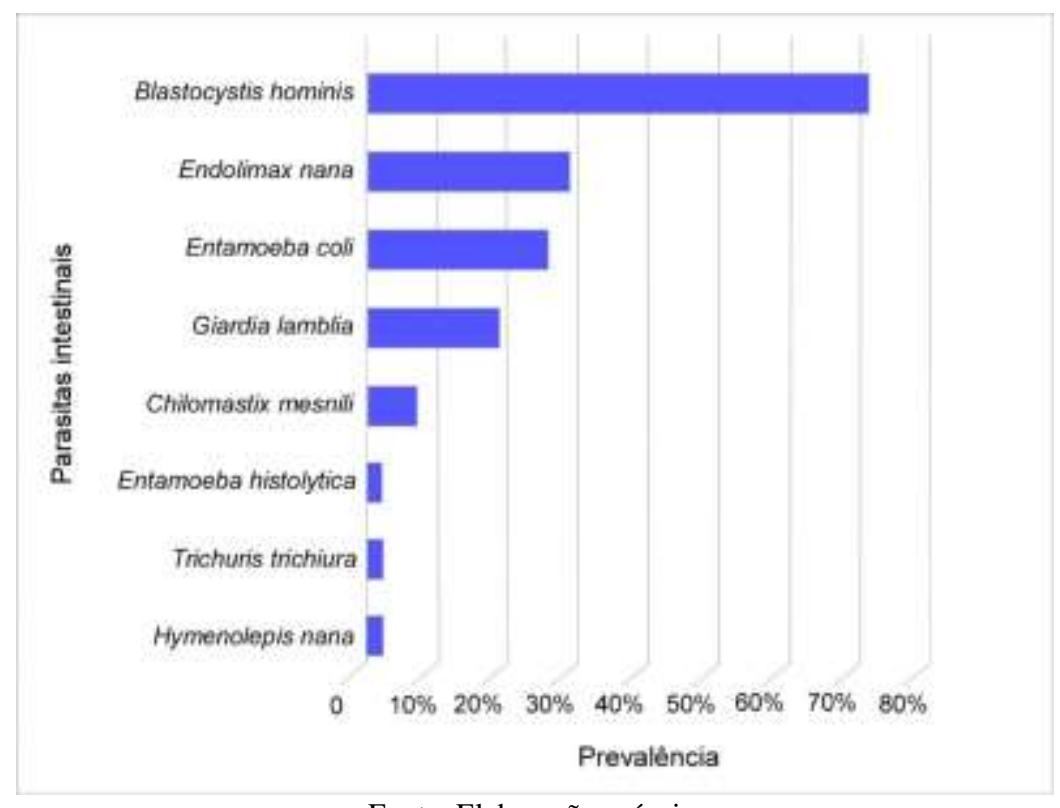

Fonte: Elaboração própria.

O parasita mais prevalente foi Blastocystis hominis, um protozoário sem flagelo encontrado no trato gastrointestinal de humanos e em outros tipos de animais, a sua transmissão é fecal-oral (Zanetti et al., 2020; Stensvold et al., 2009). Blastocystis hominis é reconhecido como um dos parasitas intestinais mais prevalentes em crianças, considerado um parasita com grande distribuição e uma prevalência muito superior à de outros parasitas intestinais. (Zorzan et al., 2020). De acordo com estudos, a sua positividade na população se deve a fatores de riscos como o consumo de água não tratada e cuidados com os animais. (Alencar et al., 2020). Pode causar diarreia e outros sintomas, porém há debates contínuos sobre sua natureza comensal ou patogênica, pois nem todos os subtipos são patogênicos, além disso, não são todos humanos suscetíveis a patogenia desse parasita. (Zanetti et al., 2020; Malheiros et al., 2011).

O protozoário intestinal Giardia lamblia, com $19 \%$ de prevalência nas crianças analisadas nesta pesquisa, é causador de doenças diarreicas agudas e crônicas, sendo frequentes em crianças (Almeida, 2019). A infeção por G. lamblia ocorrem com respectiva ingestão de cistos, através do contato direto pessoa-pessoa, pessoa-animal, ou através da ingestão de água ou alimentos contaminados. (Almeida, 2019).

Segundo Silva (2017) a infecção por Giardia lamblia foi frequentemente relatada em 40,8\% dos estudos parasitológicos no Brasil, sendo mais de $65 \%$ dos trabalhos apresentando prevalência de 0,9\% a 19,2\% de G. lamblia.

A presença de comensais como E. nana (29\%), E. coli (24\%) e C. mesnili (7\%), são indicadores a um alto grau de contaminação de água e alimentos, favorecendo a disseminação de organismos patogênicos e não patogênicos, já que apresentam as mesmas vias de transmissão. (Sena et al., 2020).

A baixa prevalência de T. trichiura e Hymenolepis nana no estudo está associado, devido aos ovos terem baixa resistência a perda de umidade e não tolerarem temperaturas fora da faixa dos $20^{\circ}-30^{\circ} \mathrm{C}$ (Oliveira et al., 2021). Em seus estudos Cardoso et al. (2020) relataram a baixa infecção de Helmintos associando a temperaturas elevadas do solo e umidade baixa, dificultando desenvolvimento dos geohelmintos, causadores das helmintíases. Tal afirmativa é confirmada pela pesquisa de Andrade et al. (2017), com crianças realizada na cidade de Campo Mourão-PR, onde houve uma baixa prevalência de Helmintos com um único helminto encontrado. Almeida et al. (2020) relatou baixo índice de infecção por Helmintos feita no munícipio de São Mateus, sendo T. trichiura (1,89\%), Hymenolepis nana (1,89\%) e o Ancilostomídeo (1,89\%), alegando a 
baixa distribuição geográfica de T. trichiura e Hymenolepis nana à alta sensibilidade dos ovos dos parasitos.

\subsection{Associação parasitária}

Analisando a frequência de múltiplas infecções de enteroparasitas nas amostras analisadas, verificou-se uma alta frequência de amostras monoparasitadas, sendo identificados 57\% de amostras monoparasitadas e $43 \%$ de amostras foram poliparasitadas (Figura 5). Resultados semelhantes foram encontrados em outros estudos, por Silva et al. (2016), Pereira et al. (2018) e Moreira et al. (2020), nos quais também identificaram em crianças maior prevalência de monoparasitismo.

As principais associações parasitárias foram as de Blastocystis hominis e Endolimax nana, tais resultados demonstram que a presença destas duas infecções está associada às condições ambientais e sanitárias insuficiente, estando relacionados com a mesma rota de contaminação entre os Protozoários. (Alencar et al., 2020; Segui et al., 2017).

Figura 5: Prevalência de múltiplas infecções por parasitas intestinais observados nas amostras da comunidade Paiol, Cáceres Mato Grosso.

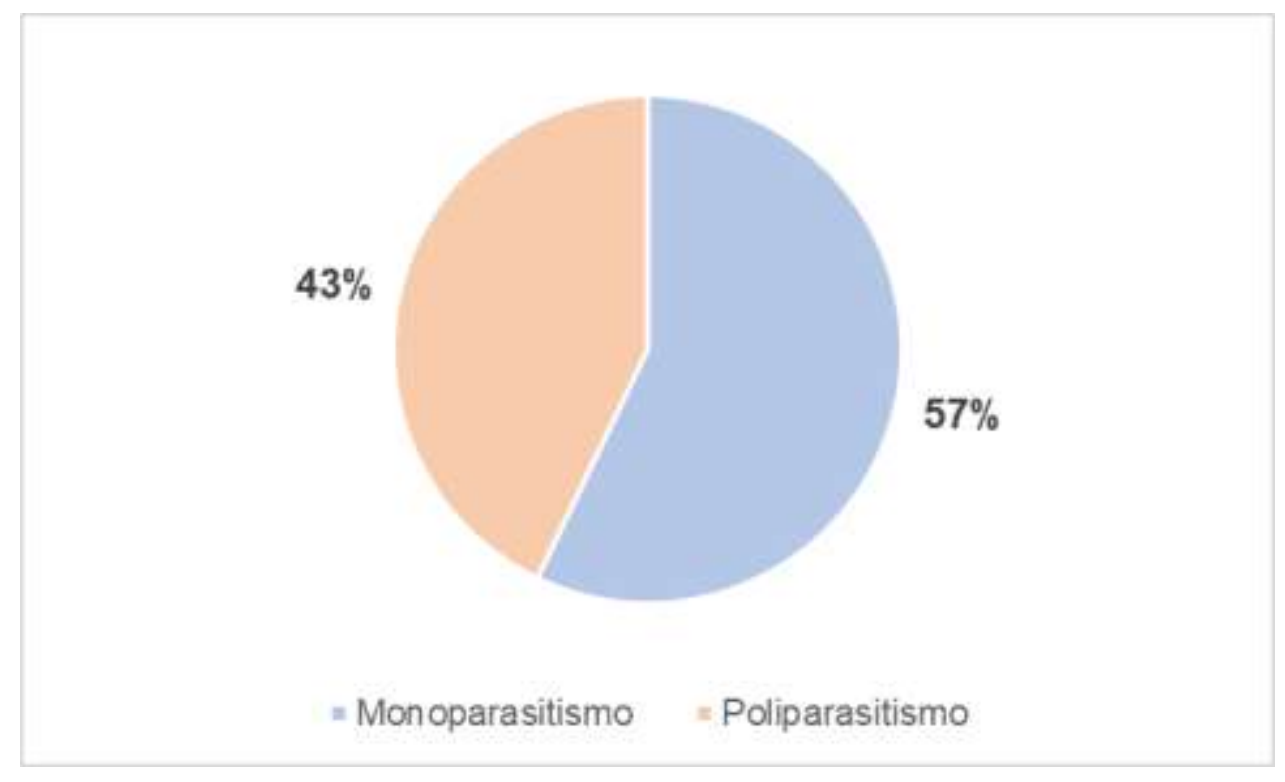

Fonte: Elaboração própria.

\subsection{Perfil sóciodemográfico, ambiental e de higiene das crianças da comunidade rural Paiol}

$\mathrm{O}$ inquérito epidemiológico das crianças da comunidade permitiu identificar fatores relativos à higiene e relações sociais e ambientais a que estão expostas (Tabela 1).

A faixa de renda dos entrevistados, variou de um a três salários mínimos. A prevalência de enteroparasitas foi maior nas faixas de renda até um salário mínimo, considerando grupos com maiores números de crianças analisadas.

De acordo com os resultados nessa pesquisa, a taxa de adesão e retorno foi de 50\%, considerado abaixo do esperado, uma vez que os exames eram gratuitos e os participantes não precisavam se locomover ao perímetro urbano. Entretanto um estudo parasitológico em escolares realizado por Souza et al. (2019) na escola Municipal São José, localizada no munícipio de Caxias do estado do Maranhão observou apenas $71,4 \%$ de participação na pesquisa. Silva et al. (2017) em estudo de prevalência em uma escola no município de Monte Carlos no estado de Minas gerais demostrou apenas 28 (46,6\%) de participação, justificando a não participação total devido a não autorização dos pais. Esses números demonstram a baixa relação e integração dos pais com as atividades envolvidas com seus filhos na escola, o que pode estar relacionado às extensas jornadas de trabalho, impedindo a participação nos projetos e negligenciamento das doenças infecto parasitárias, além de estar mais associada a classes econômicas de menor aquisição. (Oliveira et al., 2015; Rodrigues et al., 2019; Souza et al., 2019). 
Referente às características ambientais da Comunidade Paiol, 65\% dos participantes referiram possuir animais de estimação e $11 \%$ não possuem. Quanto ao material da casa, 49\% são de alvenaria e 17\% de madeira. Quanto ao piso da casa, $36 \%$ possuem piso do tipo cerâmica e $31 \%$ de cimento; $66 \%$ o quintal é de terra e $7 \%$ de grama; $46 \%$ da água é de cisterna/poço e $12 \%$ de rio/córrego; $29 \%$ não faz nenhum tratamento de água antes do consumo e $15 \%$ é feito pelo filtro de barro; 37\% usa apenas água para a higienização das frutas e legumes antes do consumo e $8 \%$ usa vinagre. Os escoadores de esgoto são feitos por fossa em $63 \%$ e $2 \%$ em rio/lago ou possui rede de esgoto. Dos entrevistados, $56 \%$ não tem coleta pública de lixo e $60 \%$ queimam o seu lixo doméstico. Observando essas variáveis, todas apresentaram altos valores na prevalência de parasitas intestinais, levando em consideração a população total analisada.

As crianças possuem animais domésticos e esse fator é muito relevante já que muitos animais, através de suas fezes, constituem uma significativa fonte de infecção parasitária (Lima, Malheiros, 2017; Zanetti et al., 2019; Zanetti et al., 2021a; 2021b), e podem ser os responsáveis pela contaminação do ambiente e infecção e reinfecção das crianças. Foi possível observar na comunidade Paiol, muitos animais, cães e gatos, transitando livremente nos espaços públicos e de acesso às crianças, como no campo de futebol, área da escola e nas ruas da comunidade.

De acordo com Neves et al. (2016), alguns protozoários patogênicos como a G. lamblia e E. histolytica, encontrados em nossos resultados, são transmitidos pela água consumida e podem resistir até a ação do cloro adicionado à água tratada.

O município de Cáceres - MT possui um precário sistema de esgotamento sanitário o que vem gerando o aumento de construção de sistema individual principalmente fossas rudimentares. Essa alternativa é mais barata, porém, é a menos segura, trazendo impactos sobre o ambiente e sobre a saúde dos moradores, sendo uma das principais fontes de contaminação do solo e da água com efeitos nocivos à saúde devido a presença de microrganismo patogênicos (Trata-Brasil, 2018). A comunidade Paiol, que é relativamente nova com 24 anos, também possuí, predominantemente, sistemas individuais de fossas rudimentares. Foi possível observar valas de descarte de água/esgoto a céu aberto ligadas a várias casas, dispostas em locais de acesso livre e frequentes de crianças da comunidade.

Quando observado os hábitos de higiene, 60\% referem sempre lavar as mãos após o uso do banheiro e $11 \%$ às vezes; $48 \%$ sempre lavam as mãos após o contato com animais e $24 \%$ às vezes; $64 \%$ sempre lavam as mãos antes de preparar os alimentos e 7\% às vezes; $58 \%$ sempre lavam as mãos antes de alimentarem-se e $11 \%$ às vezes. Essas variáveis apresentaram valores similares na prevalência de parasitas intestinais. É importante destacar que nos dias de "visita" à comunidade, não observamos nenhum sabão disponível nos banheiros da escola, o que nos leva a deduzir que a porcentagem das crianças que referem lavar as mãos após o uso do banheiro e antes da alimentação, pode estar superestimada, pois, essas crianças não higienizaram as mãos adequadamente.

Ainda que não foi possível comprovar no estudo relações estatisticamente significativas entre prevalências das parasitoses e as variáveis sóciodemográficas, ambientais e de higiene, é possível verificar a alta contaminação das crianças por parasitas intestinais, e pessoas parasitadas sem tratamento disseminam as enteroparasitoses no meio do ambiente, como solos e ambientes aquáticos. O ambiente rural apresenta condições desfavoráveis, como inexistência de pavimentação, saneamento básico precário, ausência de coleta de lixo e poluição ambiental por esgotos a céu aberto e depósitos de lixo. Essas condições aliadas ao precário e até inexistente tratamento da água para consumo, higienização dos alimentos e falta de educação higiênico-sanitária, permite o ciclo da infecção dos enteroparasitas encontrados em nosso estudo. Então indivíduos residentes em povoados isolados, tais como comunidades rurais, povoados e assentamentos, estão entre os mais vulneráveis às contaminações parasitárias, pois nem sempre é feito o diagnóstico, prevenção e tratamento adequado. (Viana et al., 2017).

A promoção de educação em saúde é o principal fator para ações de combate e prevenção contra parasitas intestinais, pois o desconhecimento a respeito das medidas preventivas é condicionante para a disseminação das enteroparasitoses (Camello et al., 2016). Os resultados dessa pesquisa evidenciam a necessidade de ampliar e intensificar o acesso à educação 
sanitária nas escolas de educação infantil, principalmente de comunidades rurais e reforçar as informações sobre os hábitos de higiene e autocuidado para uma melhora na qualidade de vida das crianças nesses ambientes.

Tabela 1. Dados sóciodemográficos, ambientais e de higiene das crianças da comunidade Paiol e a prevalência de parasitas intestinais em cada variável.

\begin{tabular}{|c|c|c|}
\hline Características & $\begin{array}{c}\text { População } \\
\text { analisada } \\
\text { N }(\%) \\
\end{array}$ & $\begin{array}{c}\text { Prevalência de parasitas } \\
\text { intestinais } \\
\mathrm{N}(\%)\end{array}$ \\
\hline \multicolumn{3}{|c|}{ Características sóciodemográficas } \\
\hline \multicolumn{3}{|l|}{ Sexo } \\
\hline Masculino & $45(60 \%)$ & $23(55 \%)$ \\
\hline Feminino & $30(40 \%)$ & $19(45 \%)$ \\
\hline \multicolumn{3}{|l|}{ Renda } \\
\hline Menos de um salário & $14(19 \%)$ & $10(71 \%)$ \\
\hline Um salário mínimo & $28(37 \%)$ & $11(39 \%)$ \\
\hline Um a dois salários mínimos & $9(12 \%)$ & $5(56 \%)$ \\
\hline Dois a três salários mínimos & $2(3 \%)$ & $2(100 \%)$ \\
\hline Não declarou & $22(29 \%)$ & $14(64 \%)$ \\
\hline \multicolumn{3}{|c|}{ Características ambientais } \\
\hline \multicolumn{3}{|l|}{ Possui animal de estimação } \\
\hline Sim & $49(65 \%)$ & $28(57 \%)$ \\
\hline Não & $8(11 \%)$ & $7(88 \%)$ \\
\hline Não declarou & $18(24 \%)$ & $11(61 \%)$ \\
\hline \multicolumn{3}{|l|}{ Moradores em casa } \\
\hline 2 a 3 & $8(11 \%)$ & $5(63 \%)$ \\
\hline 4 a 5 & $24(31 \%)$ & $12(50 \%)$ \\
\hline 6 a 7 & $21(28 \%)$ & $12(57 \%)$ \\
\hline 8 e 12 & $5(7 \%)$ & $4(80 \%)$ \\
\hline Não declarou & $17(23 \%)$ & $11(65 \%)$ \\
\hline \multicolumn{3}{|l|}{ Moradia } \\
\hline Própria & $36(48 \%)$ & $20(56 \%)$ \\
\hline Alugada & $5(7 \%)$ & $4(80 \%)$ \\
\hline Cedida & $13(17 \%)$ & $6(46 \%)$ \\
\hline Não declarou & $21(28 \%)$ & $14(67 \%)$ \\
\hline \multicolumn{3}{|l|}{ Material da casa } \\
\hline Alvenaria & $37(49 \%)$ & $18(49 \%)$ \\
\hline Madeira & $12(17 \%)$ & $9(75 \%)$ \\
\hline Adobe & $4(5 \%)$ & $2(50 \%)$ \\
\hline Não declarou & $22(29 \%)$ & $14(64 \%)$ \\
\hline \multicolumn{3}{|l|}{ Tipo de piso } \\
\hline Cimento & $24(31 \%)$ & $14(61 \%)$ \\
\hline Cerâmica & $26(36 \%)$ & $14(54 \%)$ \\
\hline Chão batido & $3(4 \%)$ & $2(67 \%)$ \\
\hline Madeira & $1(1 \%)$ & $1(100 \%)$ \\
\hline Não declarou & $21(28 \%)$ & $14(67 \%)$ \\
\hline \multicolumn{3}{|l|}{ Quintal } \\
\hline Grama & $5(7 \%)$ & $2(40 \%)$ \\
\hline Terra & $50(66 \%)$ & $29(58 \%)$ \\
\hline Cimento & $2(3 \%)$ & $1(50 \%)$ \\
\hline Não declarou & $18(24 \%)$ & $11(61 \%)$ \\
\hline \multicolumn{3}{|l|}{ Origem da água } \\
\hline Cisterna/poço & $35(46 \%)$ & $19(54 \%)$ \\
\hline Rio/Córrego & $9(12 \%)$ & $5(56 \%)$ \\
\hline Empresa de tratamento de água & $8(11 \%)$ & $6(75 \%)$ \\
\hline Não declarou & $23(31 \%)$ & $13(57 \%)$ \\
\hline \multicolumn{3}{|l|}{ Tratamento antes do consumo } \\
\hline Filtro de barro & $11(15 \%)$ & $6(55 \%)$ \\
\hline Cloro & $10(13 \%)$ & $7(70 \%)$ \\
\hline Filtro c/vela de torneira & $7(8 \%)$ & $3(44 \%)$ \\
\hline Nenhum & $22(29 \%)$ & $12(55 \%)$ \\
\hline Fervura & $1(1 \%)$ & 0 \\
\hline Não declarou & $24(32 \%)$ & $15(63 \%)$ \\
\hline \multicolumn{3}{|c|}{ Tratamento das frutas e verduras antes do consumo } \\
\hline Com água & $28(37 \%)$ & $18(64 \%)$ \\
\hline Água sanitária & $2(3 \%)$ & 0 \\
\hline
\end{tabular}




\begin{tabular}{|c|c|c|}
\hline Água e sabão & $4(5 \%)$ & 0 \\
\hline Vinagre & $6(8 \%)$ & $5(83 \%)$ \\
\hline Não declarou & $35(47 \%)$ & $18(51 \%)$ \\
\hline \multicolumn{3}{|l|}{ Escoador de esgoto } \\
\hline Fossa & $48(63 \%)$ & $28(58 \%)$ \\
\hline Vala/Rio/Lago & $2(3 \%)$ & 0 \\
\hline Rede Geral & $2(3 \%)$ & 0 \\
\hline Não declarou & $23(31 \%)$ & $14(61 \%)$ \\
\hline \multicolumn{3}{|l|}{ Coleta de lixo } \\
\hline $3 x$ & $1(1 \%)$ & 0 \\
\hline $2 \mathrm{x}$ & $3(4 \%)$ & $1(33 \%)$ \\
\hline $1 \mathrm{x}$ & $4(5 \%)$ & $1(25 \%)$ \\
\hline Não há & $42(56 \%)$ & $26(62 \%)$ \\
\hline Não declarou & $25(34 \%)$ & $15(60 \%)$ \\
\hline \multicolumn{3}{|l|}{ Destino do Lixo } \\
\hline Lançado em Terreno baldio & $2(3 \%)$ & 0 \\
\hline Queimado & $45(60 \%)$ & $27(60 \%)$ \\
\hline Enterrado & $6(8 \%)$ & $3(50 \%)$ \\
\hline Recolhido pela prefeitura & $1(2 \%)$ & 0 \\
\hline Não declarou & $21(27 \%)$ & $12(57 \%)$ \\
\hline \multicolumn{3}{|c|}{ Características de higiene } \\
\hline \multicolumn{3}{|l|}{ Lavagem das mãos } \\
\hline \multicolumn{3}{|l|}{ Após o banheiro } \\
\hline Sempre & $45(60 \%)$ & $25(56 \%)$ \\
\hline Às vezes & $8(11 \%)$ & $6(75 \%)$ \\
\hline Não declarou & $22(29 \%)$ & $13(59 \%)$ \\
\hline \multicolumn{3}{|l|}{ Após contato com animais } \\
\hline Sempre & $36(48 \%)$ & $20(56 \%)$ \\
\hline Às vezes & $18(24 \%)$ & $10(56 \%)$ \\
\hline Não declarou & $21(28 \%)$ & $13(62 \%)$ \\
\hline \multicolumn{3}{|c|}{ Antes de preparar os alimentos } \\
\hline Sempre & $48(64 \%)$ & $27(56 \%)$ \\
\hline Às vezes & $5(7 \%)$ & $1(20 \%)$ \\
\hline Não declarou & $22(29 \%)$ & $13(59 \%)$ \\
\hline \multicolumn{3}{|l|}{ Antes de se alimentar } \\
\hline Sempre & $44(58 \%)$ & $26(59 \%)$ \\
\hline Às vezes & $8(11 \%)$ & $4(50 \%)$ \\
\hline Não declarou & $23(31 \%)$ & $13(57 \%)$ \\
\hline
\end{tabular}

Fonte: Elaboração própria.

\section{Conclusão}

Com base nos resultados apresentados, verificou-se uma alta prevalência de parasitoses intestinais nos escolares do assentamento rural na comunidade Paiol, localizada no município de Cáceres, estado do Mato Grosso. Hábitos higiênicos sanitários relacionados à transmissão de enteroparasitas foram relatados, contribuindo para que o ciclo parasitário continue. Portanto, medidas simples como educação e saúde faz-se necessário para essa comunidade. Ensinar às crianças hábitos de higiene é ensinar toda a família, pois essas são multiplicadoras de boas práticas. Políticas públicas de combate aos enteroparasitas devem ser viabilizadas por seus governantes como por exemplo saneamento básico, garantindo uma melhor condição de vida com ausência de agentes patogênicos preveníeis, para a referida população.

\section{Referências}

Almeida, D. F. C. D. (2019). Deteção dos protozoários Giardia lamblia e Cryptosporidium sp. em saladas embaladas prontas a consumir (Doctoral dissertation, Universidade de Coimbra).

Andrade, R. C. R., Norberg, A. N., Serra-Freire, N. M., de Oliveira, J. T. M., Sanchez, F. G., de Santa Helena, A. A., \& Norberg, P. R. B. M. (2014). Prevalência de parasitos intestinais em ameríndios Kayapós do estado do Pará, Brasil. Revista Saúde Física \& Mental, 3(2), 50-58.

Andrade, A. D. O., De Sá, A. R. N., \& Bezagio, R. C. (2017). Prevalência de parasitoses intestinais em crianças de um centro municipal de educação infantil de Campo Mourão, PR/Brasil. Revista Uningá Review, 29(3).

Astudillo, O. G., \& Bava, A. J. (2017). Prevalência de parasitas intestinais no Hospital de Doenças Infecciosas "Dr. Francisco Javier Muñiz". Acta Bioquímica Clínica Latinoamericana, 51 (4), 681-686. 
Barbosa, Júlia Amaral et al. Análise do perfil socioeconômico e da prevalência de enteroparasitoses em crianças com idade escolar em um município de Minas Gerais. HU Revista, 43(4), 391-397.

Brasil, Ministério das Minas e Energia Secretária-geral (1982). Folha SD. 21 Cuiaba: geologia, geomorfologia, pedologia, vegetação, uso potencial da terra / Projeto RADAMBRASIL. O Projeto. https://biblioteca.ibge.gov.br/visualizacao/livros/liv13269.pdf

Brasil, T., Mundo, E., Das, S., País, D., Futuro, U., \& Internações, G. (2010). Esgotamento sanitário inadequado e impactos na saúde da população. Trata Brasil.

Bordalo, A. A. (2006). Estudo transversal e/ou longitudinal. Revista Paraense de Medicina, 20(4), 5.

Camello, J. T., Cavagnolli, N. I., Dalla Santa Spada, P. K., Poeta, J., \& Rodrigues, A. D. (2016). Prevalência de parasitoses intestinais e condições de saneamento básico das moradias em escolares da zona urbana de Caxias do Sul, Rio Grande do Sul. Scientia Medica, 26(1), ID21716-ID21716.

Cardoso, A. B., Sousa, E. A., Silva, G. D., Campelo, P. N. G., Mendes, J. R., da Silva Ventura, M. C., \& Freire, S. M. (2020). Perfil epidemiológicosocioeconômico de enteroparasitoses em crianças de 03 A 10 anos em Teresina-PI. Brazilian Journal of Development, 6(3), $11160-11175$.

Cognialli, R. C. R. Avaliação do setor de parasitologia e desempenho nos dignósticos coproparasitológicos de laboratórios de análises clínicas de Curitiba e Região Metropolitana-Paraná.

Fonseca, R. E. P. D., Barbosa, M. C. R., \& Ferreira, B. R. (2017). High prevalence of enteroparasites in children from Ribeirão Preto, São Paulo, Brazil. Revista brasileira de enfermagem, 70, 566-571.

Girardi, E. P., \& Fernandes, B. M. (2008). A luta pela terra e a política de assentamentos rurais no Brasil: a Reforma Agrária conservadora. Agrária (São Paulo. Online), (8), 73-98.

Hochman, B., Nahas, F. X., Oliveira Filho, R. S. D., \& Ferreira, L. M. (2005). Desenhos de pesquisa. Acta Cirúrgica Brasileira, $20,2-9$.

Hoffman, W. A., Pons, J. A., \& Janer, J. L. (1934). O método de sedimentação-concentração na esquistossomose mansoni.

INCRA. Instituto Nacional de Colonização e Reforma Agrária (2021) https://www.gov.br/incra/pt-br/assuntos/reforma-agraria/assentamentos.

Júnior, S. (2019). Associação entre fatores socioambientais e à ocorrência de enteroparasitoses em crianças cadastradas em uma obra social.

Lima Rosales, T. F., \& Malheiros, A. F. (2017). Contaminação Ambiental por enteroparasitas presentes em fezes de cães em uma região do Pantanal. $O$ Mundo da Saúde, 41(03), 368-377.

Malheiros, A. F., Stensvold, C. R., Clark, C. G., Braga, G. B., \& Shaw, J. J. (2011). Molecular characterization of Blastocystis obtained from members of the indigenous Tapirapé ethnic group from the Brazilian Amazon region, Brazil. The American journal of tropical medicine and hygiene, 85(6), 1050.

Menezes, R. A. O., Gomes, M. S. M., Barbosa, F. H. F., Machado, R. L. D., Andrade, R. F., \& Couto, A. A. R. D. (2013). Sensibilidade de métodos parasitológicos para o diagnóstico das enteroparasitoses em Macapá-Amapá, Brasil. Rev Biol Ciênc Terra, 13(2), 66-73.

Muñoz-Antoli, C., Gozalbo, M., Pavón, A., Pérez, P., Toledo, R., \& Esteban, J. G. (2018). Enteroparasites in preschool children on the pacific region of Nicaragua. The American journal of tropical medicine and hygiene, 98(2), 570.

Mergulhão, N. L. O. N., dos Santos, A. C. M., da Silveira Santos, S. S. G., da Silva, E. M. G., Júnior, M. C. F. L., \& Couto, J. L. A. (2020). Ocorrência de enteroparasitoses em moradores de um povoado na zona periurbana do município de Pilar (Alagoas, Brasil). Brazilian Journal of Development, 6(12), 9625596266.

Oliveira Barbosa, J., de Souza, Á. D., \& Thomé, M. P. M. (2015). Ocorrência de enteroparasitoses em alunos da escola municipalizada deputado Salim Simão em Santo Antônio de Pádua (RJ). REINPEC-Revista Interdisciplinar Pensamento Científico, 1(1).

Oliveira, A. S. D. S. S., da Silva, B. E. S., Cunha, E. A., Macedo, J. L., Rodrigues, A. C. E., Assunção, M. D. J. S. M., \& de Azevêdo, C. A. S. (2019). Enteroparasitoses em escolares da rede pública municipal. Research, Society and Development, 8(4), e384955-e384955.

Oliveira Pereira, S., Feitosa, R. B., de Carvalho Meireles, M. A., Miguel, P. S. B., da Motta, O. J. R., da Silva Boroni, F., \& Santana, L. A. (2021). TRICURÍASE. Revista Científica UNIFAGOC-Saúde, 6(1), 65-72.

OMS - Organização Mundial de Saúde. Intestinal worms. OMS, (2013). http://www.who.int/intestinal_worms/en.

Rodrigues, M. R., Martins, A. A., Martins, J. F., Oliveira, O. S., Pereira, T. F., \& Mendes, R. F. (2019). Estudo da prevalência de parasitoses em creche de Manhuaçu/MG. Anais do Seminário Científico do UNIFACIG, (5).

Santos, P. H. S., Barros, R. D. C. S., Gomes, K. V. G., Nery, A. A., \& Casotti, C. A. (2017). Prevalência de parasitoses intestinais e fatores associados em idosos. Revista Brasileira de Geriatria e Gerontologia, 20, 244-253.

Santos Zanetti, A., da Silva, B. L. S., de Barros, L. F., Garcia, H. A., de Aguiar, D. M., Espinosa, O. A., \& Malheiros, A. F. (2021). Investigação epidemiológica de parasitas gastrointestinais em cães de diferentes ambientes da região central do Brasil: implicações para a saúde humana-animalambiental. Research, Society and Development, 10(1), e31210111751-e31210111751.

Santos Zanetti, A., da Silva Junior, I. C., de Barros, L. F., Domínguez, O. A. E., Lima, G. S., de Souza Silva, A., \& Malheiros, A. F. (2019). Parasitas intestinais em cães provenientes dos biomas do nordeste brasileiro: aspecto zoonótico e ambiental. Revista Ibero-Americana de Ciências Ambientais, 10(3), 42-51. 
Research, Society and Development, v. 10, n. 16, e96101623551, 2021

(CC BY 4.0) | ISSN 2525-3409 | DOI: http://dx.doi.org/10.33448/rsd-v10i16.23551

Silva, P. V. D., Maciel, L. D. S., Castro, L. S., Murat, P. G., Higa Junior, M. G., Zerlotti, P. H., \& Dorval, M. E. C. (2018). Enteroparasites in riverside settlements in the Pantanal wetlands ecosystem. Journal of parasitology research, 2018.

Silva Miranda, M. R., da Silva Luz, C. C., da Silva Neves, S. M. A., \& Scheuer, J. M. (2018). Cobertura vegetal e uso da terra no Assentamento Paiol-Brasil: Subsídios Para a Conservação do Pantanal. Revista de Geografia-PPGEO-UFJF, 8(1).

Silva, M. F. F. D. (2017). Caracterização de genótipos de Giardia lamblia e ferramentas de educação em saúde como estratégias de prevenção da giardíase (Doctoral dissertation).

Silva, P. L. N., Cerqueira, F. A., Ferraz, R. S., Vaz, M. D. T., \& Fonseca, J. R. (2017). Análise da prevalência parasitológica em amostras fecais de crianças de uma escola da rede pública do estado de minas gerais. Revista Contexto \& Saúde, 17(33), 146-154.

Silva Neves, S. M. A., Nunes, M. C. M., \& Neves, R. J. (2011). Caracterização das condições climáticas de Cáceres/MT-Brasil, no período de 1971 a 2009: subsídio às atividades agropecuárias e turísticas municipais. Boletim goiano de geografia, 31(2), 55-68.

Stensvold, C. R., Lewis, H. C., Hammerum, A. M., Porsbo, L. J., Nielsen, S. S., Olsen, K. E. P., \& Mølbak, K. (2009). Blastocystis: unravelling potential risk factors and clinical significance of a common but neglected parasite. Epidemiology \& Infection, 137(11), 1655-1663.

Souza, J. B. D. (2018). Prevalência de enteroparasitoses em crianças matriculadas nas creches do município de Cruz das Almas-Ba.

Sousa, F. D. C. A., Siqueira, H. D. S., Siqueira, F. F. F. S., de Oliveiira, E. H., Verde, R. M. C. L., Junior, R. N. C. M., \& Maia, N. M. S. (2019). Prevalência de parasitoses intestinais em crianças de uma escola pública municipal. Revista Enfermagem Atual In Derme, 90(28).

Viana, M. L., Fialho, N. R., Rocha, S. M. S., Alves, T. C. L. A., da Trindade, R. A., \& Melo, A. C. F. L. (2017). Parasitoses intestinais e a inter-relação com os aspectos socioeconômicos de indivíduos residentes em um povoado rural (Rosápolis de Parnaíba-PI). Scientia Plena, 13(8).

Zanetti, A. S., de Barros, L. F., de Araújo, M. S., Garcia, H. A., Aguiar, D. M., Espinosa, O. A., \& Malheiros, A. F. (2021). Diversity and prevalence of intestinal parasites of zoonotic potential in animal hosts from different biomes in the central region of Brazil. Annals of Parasitology, 67(1), 95-105.

Zanotto, M., Cavagnolli, N. I., Breda, J. C., Dalla Santa Spada, P. K. W., Bortolini, G. V., \& Rodrigues, A. D. (2018). Prevalência de parasitoses intestinais e avaliação socioeconômica em município da região da Serra Gaúcha, Rio Grande do Sul, Brasil. Revista de Patologia Tropical / Journal of Tropical Pathology, 47 (1), 19-30.

Zorzan, W. N. M., Moreira, L. M., da Silva, S. L., Castrillon, S. K. I., Lima, M. F., da Silva, M. E. V., \& Francisco, A. Prevalência de Blastocystis spp. provenientes de amostras fecais de moradores de dois biomas mato-grossenses. 